\title{
A Planning Method for Energy Storage System of Integrated Community Energy System Considering Demand Response
}

\author{
Jinghua $\mathrm{Li}^{1}$, Chenbing Hua ${ }^{1}$, Deyu Jiang ${ }^{1}$, Qian Jiang ${ }^{2, *}$, and Kuihua $\mathrm{Wu}^{3}$ \\ ${ }^{1}$ State Grid Linyi Power Supply Company, Linyi 276003, Shandong Province, China \\ ${ }^{2}$ Key Laboratory of Smart Energy \& Information Technology of Tianjin Municipality (Tianjin University), Nankai District, Tianjin \\ ${ }^{3}$ Economic \& Technology Research Institute of State Grid Shandong Electric Power Company, Jinan 2500211, Shandong Province, China
}

\begin{abstract}
Demand response plays a significant role in peak load shifting, storage capacity configuration and renewable energy utilization. A bi-level planning method for energy storage system of integrated community energy system considering the demand response is proposed in this paper. In the upper level, the investment cost of electrical energy storage and thermal energy storage, operation and maintenance cost and fuel cost of the integrated community energy system, as well as the compensation cost to the energy consumer, are considered; in the lower level, the responded demand of the energy consumer is taken into consideration to minimize the energy bill of the energy consumer. An actual planning for energy storage system of integrated community energy system shows the effectiveness of the proposed method.
\end{abstract}

\section{Introduction}

The increasing focus on the efficiency of energy sectors and the reduction of greenhouse gas emission in recent years has aroused attention on the integrated energy system (IES) [1]. With the development of the distributed generation and energy conversion devices, the energy supply system is further closer to the energy consumers $(\mathrm{ECs})[2,3]$. Therefore, as one of the main application forms of IES, the integrated community energy system (ICES) has been rapidly constructed and developed.

Generally, the energy server (ES) is responsible for the planning and operation of ICES. The ES purchases electricity and natural gas from public utilities. The EC purchases electricity and heating energy from the ES. Energy storage system (ESS) plays an important part for both ES and EC in ICES. the promotion of renewable energy utilization [4] and ancillary services [5] could be realized by the installation of the ESS, so the economic benefit of the ES is increased. As for the EC, the ESS guarantees the energy supplying reliability [6]. It is of great importance to make an optimal planning scheme of the ESS in ICES.

Extensive efforts have been made to study the optimal planning of the ESS. Uncertainties [7], reliability [6] and life of ESS [8] have been taken into consideration during the ESS capacity configuration. However, the impact of the demand response (DR) of the EC on the ESS planning scheme has not been fully investigated.

DR comprises incentive-based programs and pricebased programs (time-of-use, critical peak pricing, dynamic pricing, etc.) $[9,10]$. The DR applicated in utilities have been proved to be a potential way to benefit all the participants [11]. A complexity algorithm in [12] outlined the cooperation between the DR and ESS in operation stage. Besides, the advantage of DR in thermal energy storage (TES) management shows the prospect of the DR in ICES.

To fully investigate the impact of the DR on the ESS planning scheme, a bi-level planning method for ESS of ICES considering the demand response of the EC is proposed in this paper. In the proposed method, the investment cost of electrical energy storage (EES) and thermal energy storage (TES), operation and maintenance cost and fuel cost of the ICES as well as the compensation cost to the EC are considered in the upper level; The responded demand of the $\mathrm{EC}$ is taken into consideration in the lower level to minimize the annual energy bill of the EC.

\section{The framework of the bi-level planning method}

The diagram of the proposed bi-level planning method is shown in Fig. 1.

The upper level represents the ESS planning and optimization problem. The ES optimizes the capacity and operation strategy of ESS with the minimum annual cost as its goal.

The lower level represents the optimization problem of demand response strategy of the EC. The EC optimizes the consuming strategy according to the energy price made by the ES with the minimum annual energy bill.

\footnotetext{
*Corresponding author: qjiang@tju.edu.cn
} 


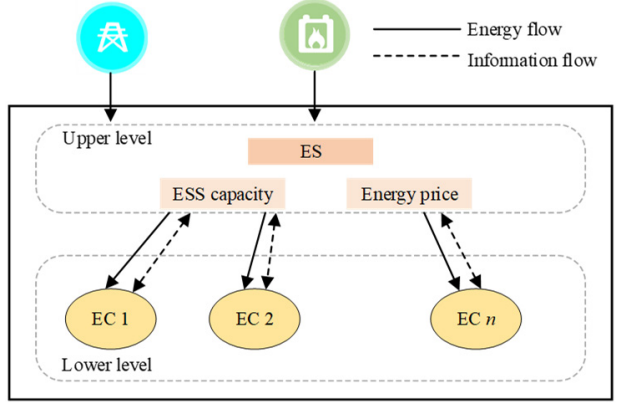

Fig. 1 framework of the bi-level planning method

\section{Model of the ICES}

\subsection{Model of the ESS}

The charging and discharging power of electricity storage system (ESS) and heating storage system (HSS) can be continuously adjusted within a certain range. The energy storage capacity does not exceed the upper and lower limits of energy storage. The charging and discharging cannot be carried out at the same time, and the stored energy should be released in a scheduling cycle to avoid energy loss caused by long-term storage. while the constraints of the thermal energy storage (TES) are similar to those of ESS and will not be repeated.

$$
\begin{gathered}
0 \leq P_{d, t}^{\mathrm{ESS}, \mathrm{ch}} \leq \bar{P}^{\mathrm{ESS}, \mathrm{ch}} \\
0 \leq P_{d, t}^{\mathrm{ESS}, \mathrm{dis}} \leq \bar{P}^{\mathrm{ESS}, \mathrm{dis}} \\
W_{d, t+1}^{\mathrm{ESS}}=W_{d, t}^{\mathrm{ESS}}(1-\sigma)+\eta^{\mathrm{ESS}, \mathrm{ch}} P_{d, t}^{\mathrm{ESS}, \mathrm{ch}} \Delta t- \\
P_{d, t}^{\mathrm{ESS}, \mathrm{dis}} \Delta t / \eta^{\mathrm{ESS}, \mathrm{dis}}, t=1,2 \ldots T-1 \\
\underline{W}^{\mathrm{ESS}} \leq W_{d, t}^{\mathrm{ESS}} \leq \bar{W}^{\mathrm{ESS}} \\
W_{0}^{\mathrm{ESS}}=W_{T}^{\mathrm{ESS}} \\
P_{d, t}^{\mathrm{ESS}, \mathrm{ch}} P_{d, t}^{\mathrm{ESS}, \mathrm{dis}}=0
\end{gathered}
$$

where, $P_{t}^{\mathrm{ESS}, \mathrm{ch}}$ and $P_{t}^{\mathrm{ESS}, \mathrm{dis}}$ are the charging power and discharging power of ESS at the time of $t$, respectively; $\bar{P}^{\text {ESS,ch }}$ and $\bar{P}^{\text {ESS,dis }}$ are the charging upper limit and discharging upper limit of ESS, respectively; $\sigma$ is the self-discharging rate of ESS; $\eta^{\text {ESS,ch }}$ and $\eta^{\text {ESS,dis }}$ are the charging efficiency and discharging efficiency of ESS, respectively; $W_{s, t}^{\mathrm{ESS}}$ is the energy storage capacity of ESS; $\underline{W}^{\mathrm{ESS}}$ and $\bar{W}^{\mathrm{ESS}}$ are the maximum and minimum energy storage capacity of ESS, respectively.

\subsection{Model of DR}

For EC, the load can be divided into fixed load and flexible load. The fixed load is not affected by the price, while the flexible load is the transferable load that is sensitive to the energy price and can be transferred from the peak price period to the valley price period.

$$
\begin{gathered}
P_{d, t}^{\mathrm{LD}}=P_{d, t}^{\mathrm{LD}, \mathrm{fixed}}+P_{d, t}^{\mathrm{D}} \\
Q_{d, t}^{\mathrm{LD}}=Q_{d, t}^{\mathrm{LD}, \text { fixed }}+Q_{d, t}^{\mathrm{D}} \\
\underline{P}_{d, t}^{\mathrm{D}} \leq P_{d, t}^{\mathrm{D}} \leq \bar{P}_{d, t}^{\mathrm{D}}: \underline{\lambda}_{d, t}^{\mathrm{e}}, \bar{\lambda}_{d, t}^{\mathrm{e}}
\end{gathered}
$$

$$
\begin{gathered}
\underline{Q}_{d, t}^{\mathrm{D}} \leq Q_{d, t}^{\mathrm{D}} \leq \bar{Q}_{d, t}^{\mathrm{D}}: \underline{\lambda}_{d, t}^{\mathrm{h}}, \bar{\lambda}_{d, t}^{\mathrm{h}} \\
\sum_{t=1}^{T} P_{d, t}^{\mathrm{D}}=0: \rho_{d}^{\mathrm{e}} \\
\sum_{t=1}^{T} Q_{d, t}^{\mathrm{D}}=0: \rho_{d}^{\mathrm{h}}
\end{gathered}
$$

where, $P_{d, t}^{\mathrm{LD}, \mathrm{fixed}}$ and $P_{d, t}^{\mathrm{D}}$ are EC's fixed power demand and responded demand at the time of $t$ on the day of $d$, respectively; $Q_{d, t}^{\mathrm{D} \text {,fixed }}$ and $Q_{d, t}^{\mathrm{LD}, \mathrm{flex}}$ are EC's fixed heating demand and responded heating demand at the time of $t$ on the day of $d$, respectively; $\bar{P}_{d, t}^{\mathrm{D}}$ and $\underline{P}_{d, t}^{\mathrm{D}}$ are the adjustable upper and lower limits of EC's responded power demand at the time of $t$ on the day of $d$, respectively; $\bar{Q}_{d, t}^{\mathrm{D}}$ and $\underline{Q}_{d, t}^{\mathrm{D}}$ are the adjustable upper and lower limits of EC's responded heating demand at the time of $t$ on the day of $d$, respectively. In case of $P_{d, t}^{\mathrm{D}}>0\left(Q_{d, t}^{\mathrm{D}}>0\right)$, the responded demand is transferred to this period from the high-price period; in case of $P_{d, t}^{\mathrm{D}}<0\left(Q_{d, t}^{\mathrm{D}}<0\right)$, the responded demand is transferred from this period to the lower-price period. The dual variables are given after a colon.

\subsection{Model of energy conversion device}

\section{1) $\mathrm{CHP}$}

CHP generates electricity and heat by consuming natural gas. Its operation constraints are as follows:

$$
\begin{gathered}
P_{d, t}^{\mathrm{CHP}}=\eta_{\mathrm{g} 2 \mathrm{p}}^{\mathrm{CHP}} f_{d, t}^{\mathrm{CHP}} \\
Q_{d, t}^{\mathrm{CHP}}=\eta_{\mathrm{h} 2 \mathrm{p}}^{\mathrm{CHP}} P_{d, t}^{\mathrm{CHP}} \\
\underline{P}^{\mathrm{CHP}} \leq P_{d, t}^{\mathrm{CHP}} \leq \bar{P}^{\mathrm{CHP}}
\end{gathered}
$$

where, $P_{d, t}^{\mathrm{CHP}}$ and $Q_{d, t}^{\mathrm{CHP}}$ are the electricity output and heat output of CHP at the time of $t$ on the day of $d$, respectively; $\bar{P}^{\mathrm{CHP}}$ and $\underline{P}^{\mathrm{CHP}}$ are the maximum and minimum output of CHP, respectively; $f_{d, t}^{\mathrm{CHP}}$ is the natural gas consumed by CHP at the time of $t$ on the day of $d ; \eta_{\mathrm{g} 2 \mathrm{p}}^{\mathrm{CHP}}$ is the power generation efficiency of CHP; $\eta_{\mathrm{h} 2 \mathrm{p}}^{\mathrm{CHP}}$ is the heat-toelectricity ratio of $\mathrm{CHP}$.

2)Photovoltaic

Photovoltaic (PV) output is mainly affected by the solar radiation intensity.

$$
P_{d, t}^{\mathrm{PV}}=\lambda^{\mathrm{PV}} \operatorname{Cap}^{\mathrm{PV}} G_{d, t} / G_{\mathrm{STC}}
$$

where, $P_{d, t}^{\mathrm{PV}}$ is the PV output at the time of $t$ on the day of $d ; \quad \lambda^{\mathrm{PV}}$ is the PV derating factor, equals to $0.9, G_{d, t}$ is the solar radiation intensity at the time of $t$ on the day of $d$; $G_{\mathrm{STC}}$ is the solar radiation intensity under the standard testing conditions, equals to $1 \mathrm{~kW} / \mathrm{m}^{2}$.

3) Gas boiler

Gas boiler (GB) generates heat energy by consuming natural gas. Its constraints are as follows:

$$
\begin{gathered}
Q_{d, t}^{\mathrm{GB}}=\eta^{\mathrm{GB}} f_{d, t}^{\mathrm{GB}} \\
\underline{Q}^{\mathrm{GB}} \leq Q_{d, t}^{\mathrm{GB}} \leq \bar{Q}^{\mathrm{GB}}
\end{gathered}
$$


where, $Q_{d, t}^{\mathrm{GB}}$ is the heating output of GB at the time of $t$ on the day of $d ; f_{d, t}^{\mathrm{GB}}$ is the natural gas consumed by GB at the time of $t$ on the day of $d ; \eta^{\mathrm{GB}}$ is the working efficiency of GB; $\bar{Q}^{\mathrm{GB}}$ and $\underline{Q}^{\mathrm{GB}}$ are the maximum and minimum heating output of $\mathrm{GB}$, respectively.

3) Electric boiler

Electric boiler (EB) generates heat energy by consuming electricity.

$$
\begin{gathered}
Q_{d, t}^{\mathrm{EB}}=\eta^{\mathrm{EB}} P_{d, t}^{\mathrm{EB}} \\
\underline{Q}^{\mathrm{EB}} \leq Q_{d, t}^{\mathrm{EB}} \leq \bar{Q}^{\mathrm{EB}}
\end{gathered}
$$

where, $Q_{d, t}^{\mathrm{EB}}$ is the heating output of $\mathrm{EB}$ at the time of $t$ on the day of $d ; Q_{d, t}^{\mathrm{EB}}$ is the electric power consumed by EB at the time of $t$ on the day of $d ; \eta^{\mathrm{EB}}$ is the working efficiency of EB; $\bar{Q}^{\mathrm{EB}}$ and $\underline{Q}^{\mathrm{EB}}$ are the maximum and minimum heating output of $\mathrm{EB}$, respectively.

\section{ESS planning model}

\subsection{Objective function}

\subsubsection{Upper level}

The ES maximizes its annual cost, including the annualized planning cost, operation and maintenance cost of the ESS, and the compensation cost to the EC.

$$
\min C_{\mathrm{Inv}}+C_{\mathrm{OM}}+C_{\mathrm{Com}}
$$

where, $C_{\mathrm{Inv}}$ is the planning cost; $C_{\mathrm{OM}}$ is the operation and maintenance $(\mathrm{O} \& \mathrm{M})$ cost, $C_{\mathrm{Com}}$ is the compensation cost.

1) Planning cost

Planning cost is used to characterize the cost of multienergy equipment and renewable energy equipment capacity configuration. In this paper, the value of $C_{\operatorname{lnv}}$ is described by the annual planning cost.

$$
\begin{aligned}
C_{\mathrm{Inv}}= & \frac{r(1+r)^{y}}{(1+r)^{y}-1} . \\
& \sum\left(\operatorname{Cap}^{\mathrm{EES}} w^{\mathrm{EES}}+\operatorname{Cap}^{\mathrm{TES}} w^{\mathrm{TES}}\right)
\end{aligned}
$$

where, $y$ is the system planning horizon; $r$ is the capital discount rate; $C a p^{\mathrm{EES}}$ and $C a p^{\mathrm{TES}}$ are the planning capacity of ESS and TES, respectively; $w^{\mathrm{EES}}$ and $w^{\mathrm{TES}}$ are the unit planning cost of equipment $i$.

2) O\&M cost

O\&M cost consists of electricity purchase cost, gas purchase cost and equipment maintenance cost.

$$
C_{\mathrm{OM}}=\sum_{d}^{365} \sum_{t=1}^{T}\left(u_{d, t}^{\mathrm{e}} f_{d, t}^{\mathrm{e}}+u_{d, t}^{\mathrm{h}} f_{d, t}^{\mathrm{h}}\right)+\sum_{d}^{365} \sum_{t=1}^{T} \sum_{i \in \mathrm{I}} v_{i} P_{i, d, t}
$$

where, $u_{d, t}^{\mathrm{e}}$ and $u_{d, t}^{\mathrm{h}}$ are the electricity purchase price and gas purchase price of ES from EP at the time of $t$ on the day of $d$, respectively; $f_{d, t}^{\mathrm{e}}$ and $f_{d, t}^{\mathrm{h}}$ are the electricity quantity and gas quantity purchased by ES from EP at the time of $t$ on the day of $d$, respectively; $P_{i, d, t}$ is the output of equipment $i$ at the time of $t$ on the day of $d ; v_{i}$ is the unit maintenance cost of equipment $i$.

3) Compensation cost

Compensation cost is composed of the cost of the electrical and thermal deferrable load demand response.

$$
C_{\text {Com }}=\sum_{d=1}^{365} \sum_{t=1}^{T}\left(r_{d, t}^{\mathrm{ecc}} P_{d, t}^{\mathrm{D}}+r_{d, t}^{\mathrm{h}, \mathrm{c}} Q_{d, t}^{\mathrm{D}}\right)
$$

where, $r_{d, t}^{\mathrm{e}}$ and $r_{d, t}^{\mathrm{h}}$ are the compensation electricity price and heating price at the time of $t$ on the day of $d$ set by ES, respectively; $P_{d, t}^{\mathrm{D}}$ and $Q_{d, t}^{\mathrm{D}}$ are responded electricity load and heating load at the time of $t$ on the day of $d$, respectively.

\subsubsection{Lower level}

The EC adjusts its demand according to the energy price set by the ES to minimize the annual energy bill.

$$
\begin{aligned}
\min C^{\text {cost }}= & \sum_{d=1}^{365} \sum_{t=1}^{T}\left(r_{d, t}^{\mathrm{e}} P_{d, t}^{\mathrm{LD}}+r_{d, t}^{\mathrm{h}} Q_{d, t}^{\mathrm{LD}}\right. \\
& \left.-r_{d, t}^{\mathrm{ec}} P_{d, t}^{\mathrm{D}}-r_{d, t}^{\mathrm{h}, \mathrm{c}} Q_{d, t}^{\mathrm{D}}\right)
\end{aligned}
$$

where, $r_{d, t}^{\mathrm{e}}$ and $r_{d, t}^{\mathrm{h}}$ are the electricity price and heating price, respectively; $P_{d, t}^{\mathrm{LD}}$ and $Q_{d, t}^{\mathrm{LD}}$ are the electricity load and heating load, respectively.

\subsection{Constraints}

1) Pricing constraints

The compensation price set by ES should be constrained in order to guarantee the interests of the EC. In this way, the average energy price set by ES will not be higher than that of EP's energy price. The specific expressions are as follows:

$$
\begin{aligned}
& \underline{\alpha} u_{d, t}^{\mathrm{e}} \leq r_{d, t}^{\mathrm{e}}-r_{d, t}^{\mathrm{e}, \mathrm{c}} \leq \bar{\alpha} u_{d, t}^{\mathrm{e}} \\
& \frac{1}{T} \sum_{t=1}^{T}\left(r_{d, t}^{\mathrm{e}}-r_{d, t}^{\mathrm{e}, \mathrm{c}}\right) \leq \frac{1}{T} \sum_{t=1}^{T} u_{d, t}^{\mathrm{e}} \\
& \underline{\beta} u_{d, t}^{\mathrm{h}} \leq r_{d, t}^{\mathrm{h}}-r_{d, t}^{\mathrm{h}, \mathrm{c}} \leq \bar{\beta} u_{d, t}^{\mathrm{h}} \\
& \frac{1}{T} \sum_{t=1}^{T}\left(r_{d, t}^{\mathrm{h}}-r_{d, t}^{\mathrm{h}, \mathrm{c}}\right) \leq \frac{1}{T} \sum_{t=1}^{T} u_{d, t}^{\mathrm{h}}
\end{aligned}
$$

Eq. (26) and Eq. (27) describe the constraints on upper and lower limits of ES's electricity price and heating price, respectively. Where, $\underline{\alpha}$ and $\bar{\alpha}$ are the lower limit and upper limit of ES's electricity price. $\underline{\beta}$ and $\bar{\beta}$ are the lower limit and upper limit of ES's heating price, respectively. Eq. (27) and Eq. (29) describe the constraints on the average value of electricity price and heating price, respectively. Where, $u_{d, t}^{\mathrm{e}}$ and $u_{d, t}^{\mathrm{h}}$ are the EP's electricity price and heating price at the time of $t$ on the day of $d$, respectively.

2) Energy balance constraints

The electricity and heating energy balance are considered to describe the energy balance in the ICES.

$$
\begin{aligned}
P_{d, t}^{\text {grid }}+P_{d, t}^{\mathrm{PV}}+P_{d, t}^{\mathrm{CHP}}+P_{d, t}^{\mathrm{ES}, \mathrm{dis}}-P_{d, t}^{\mathrm{ES}, \mathrm{ch}}= \\
P_{d, t}^{\mathrm{LD}}-P_{d, t}^{\mathrm{LD}}+P_{d, t}^{\mathrm{EB}}
\end{aligned}
$$




$$
Q_{d, t}^{\mathrm{CHP}}+Q_{d, t}^{\mathrm{GB}}+\mathrm{Q}_{d, t}^{\mathrm{EB}}=Q_{d, t}^{\mathrm{LD}}-Q_{d, t}^{\mathrm{D}}
$$

3) Capacity constraints in

The capacity constraints of the EES and TES are stated

$$
\begin{aligned}
& 0 \leq C a p^{\mathrm{EES}} \leq \overline{\operatorname{Cap}}^{\mathrm{EES}} \\
& 0 \leq \mathrm{Cap}^{\mathrm{TES}} \leq \overline{\operatorname{Cap}}^{\mathrm{TES}}
\end{aligned}
$$

where, $\operatorname{Cap}^{\mathrm{EES}}$ and $\operatorname{Cap}^{\mathrm{TES}}$ are the capacity of the EES and TES, respectively; $\overline{C a p}^{\mathrm{EES}}$ and $\overline{\mathrm{Cap}}^{\mathrm{TES}}$ are the maximum installation capacity of the EES and TES, respectively.

Constraints (1)-(20), (26)-(33) and objective function (21) and (25) form a nonlinear bi-level planning model.

\subsection{Solution technique}

The bilinear term $r_{d, t}^{\mathrm{e}, \mathrm{c}} P_{d, t}^{\mathrm{D}}, \quad r_{d, t}^{\mathrm{h}, \mathrm{c}} Q_{d, t}^{\mathrm{D}}$, and complementary constraint (6) make it difficult to solve the planning problem. To tackle this problem, Karush-Kuhn-Tucker (KKT) conditions and strong duality theorem are utilized to convert the proposed bi-level planning model to MILP single-level programming problem.

\section{Case study}

\subsection{Test system and parameters}

An actual ICES in North China is selected as the test system, and EC is an electricity-heating coupled user., while the capacity configuration of the ESS is carried out from the perspective of ES. CHP, GB, EB, EES and TES are considered as the multi-energy equipment in the ICES; $\mathrm{PV}$ is the only renewable resource considering the distribution characteristics of renewable energy. The relevant parameters of these energy conversion devices are shown in

Tab. 1 Parameters of the energy conversion devices

\begin{tabular}{lll}
\hline Devices & Capacity & Technical parameter \\
\hline $\mathrm{CHP}$ & $1200 \mathrm{~kW}$ & $\eta_{\mathrm{g} 2 \mathrm{p}}^{\mathrm{CHP}}=0.45$ \\
& & $\eta_{\mathrm{h} 2 \mathrm{p}}^{\mathrm{CHP}}=0.7$ \\
& & $v_{\mathrm{CHP}}=0.05 ¥ / \mathrm{kWh}$ \\
$\mathrm{GB}$ & $1000 \mathrm{~kW}$ & $\eta^{\mathrm{GB}}=0.95$ \\
& & $v_{\mathrm{GB}}=0.01 ¥ / \mathrm{kWh}$ \\
$\mathrm{EB}$ & $300 \mathrm{~kW}$ & $\eta^{\mathrm{GB}}=0.9$ \\
& & $v_{\mathrm{EB}}=0.01 ¥ / \mathrm{kWh}$ \\
$\mathrm{PV}$ & $3000 \mathrm{~kW}$ & $\lambda^{\mathrm{PV}}=0.9$ \\
& & $v_{\mathrm{PV}}=0.02 ¥ / \mathrm{kWh}$ \\
\hline
\end{tabular}

Tab. 2 Parameters of the ESS

\begin{tabular}{lll}
\hline Devices & $\begin{array}{l}\text { Economic } \\
\text { parameter }\end{array}$ & $\begin{array}{l}\text { Technical } \\
\text { parameter }\end{array}$ \\
\hline $\mathrm{ESS}$ & $w_{\mathrm{ESS}}=1200 ¥ / \mathrm{kW}$ & $\eta^{\text {ESS,dis }}=0.95$ \\
\hline
\end{tabular}

\begin{tabular}{lll}
\hline & $v_{\mathrm{ESS}}=0.01 ¥ / \mathrm{kWh}$ & $\eta^{\mathrm{ESS}, \mathrm{ch}}=0.95$ \\
$\mathrm{HSS}$ & $w_{\mathrm{HSS}}=190 ¥ / \mathrm{kW}$ & $\eta^{\text {HSS,dis }}=1$ \\
& $v_{\mathrm{HSS}}=0.01 ¥ / \mathrm{kWh}$ & $\eta^{\text {HSS,ch }}=1$
\end{tabular}

Considering the actual operation of ICES in the whole year, the illumination data and load data of typical days in transition season, summer and winter are selected. The typical power/heat load curve and light intensity are shown in Fig. 2, the time-of-use price is shown in Fig. 3. The price of natural gas was $2.53 \mathrm{Y} / \mathrm{m}^{3}$, which is 0.26 $Y / \mathrm{kWh}$. The planning horizon of ES for ICES is 20 years, and the discount rate is $6 \%$.

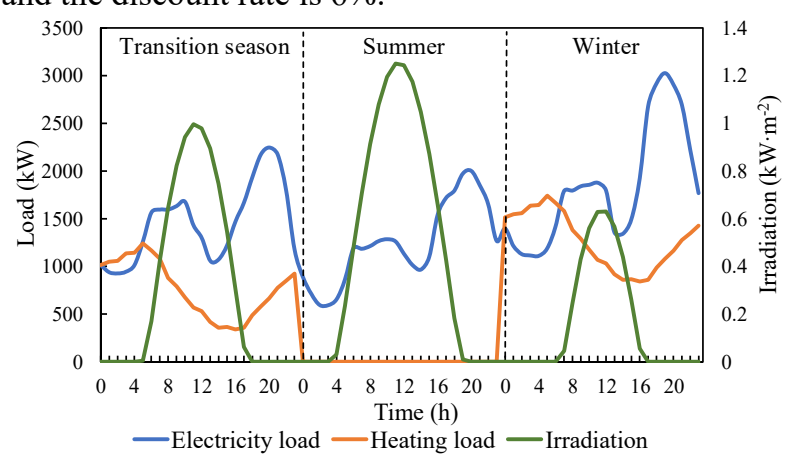

Fig. 2 Three Typical Day Data

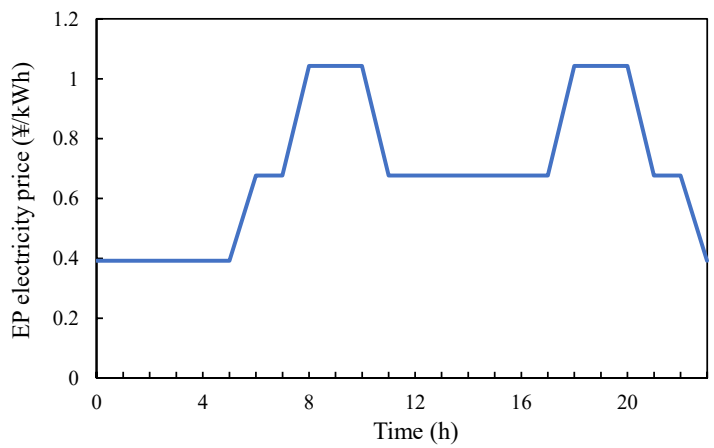

Fig. 3 time-of-use power price

In order to fully reflect the consideration of the influence of DR on ICES planning and the effectiveness of the proposed method, two cases are set for comparative analysis:

Case I: ICES planning considering the DR.

Case II: ICES planning without considering the DR.

\subsection{Planning results and analysis}

\subsubsection{Capacity of the ESS}

By solving ICES planning of Case I and Case II, the equipment capacity is obtained, as shown in

Tab. 3 planning scheme of the ESS

\begin{tabular}{ccc}
\hline Device & Case I & Case II \\
\hline EES $(\mathrm{kWh})$ & 1963 & 336 \\
TES $(\mathrm{kWh})$ & 3647 & 848 \\
\hline
\end{tabular}


In terms of equipment capacity, the capacity of EES and TES is smaller than that in Case II. Explanation of differences in the planning schemes will be given from the perspective of economy and operation performance.

\subsubsection{Economic analysis}

As shown in Fig. 4, the annual investment cost of ESS in Case I is reduced by 184,696 yuan compared with Case II, a decrease of $46.7 \%$. The annual electricity purchase cost from EP is decreased by 2,029 yuan, while the annual gas purchase cost is decreased by 59,793 yuan, and the annual maintenance cost is reduced by 20,359 yuan. As for the compensation to the EC, 253,895 yuan is paid to the EC compared with Case II. The total cost is decreased by 12,982 yuan.

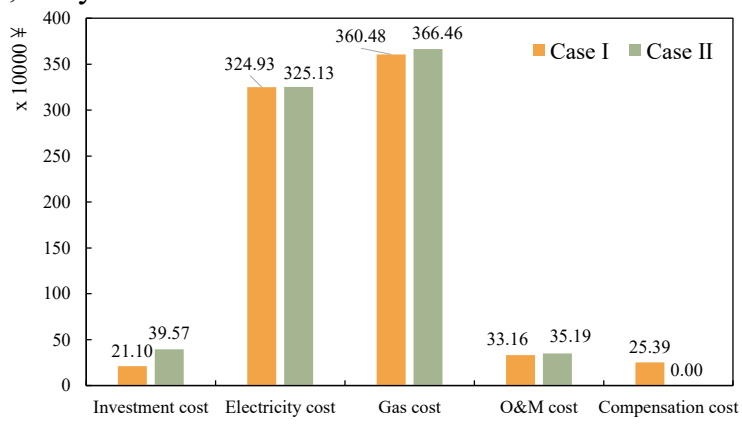

Fig. 4 Economy of the ES

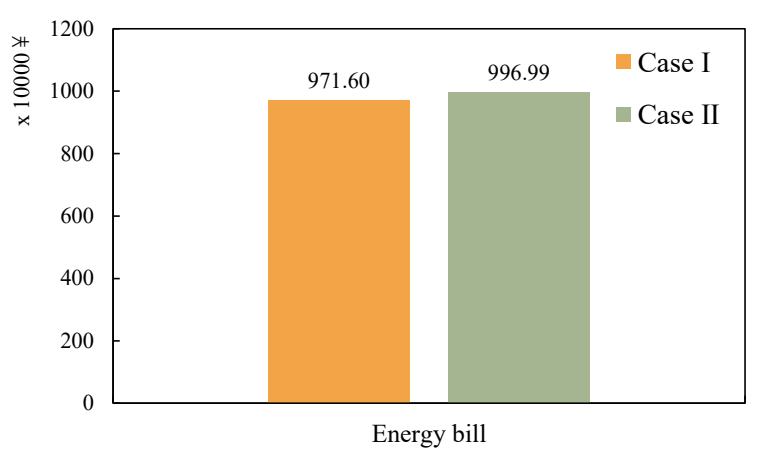

Fig. 5 Economy of the EC

Fig. 5 gives a description of the economy of the EC. Compared with Case II, EC adjusts its electricity load and heating load according to the energy price made by the ES to participate in the DR, which makes the energy bill of the EC decreased by 253,895 yuan.

\subsubsection{Operation analysis}

The electricity and heating balance of the ICES are shown in Fig. 6 and Fig. 7, respectively. It can be seen from Fig. 6 that the high-price period is set at 19:00-21:00 by ES, during which the EC transfers out its flexible load, thus making the capacity of EES decreased. ES utilizes the flexible load of EC as energy storage resource by setting an appropriate energy price, and reduces the planning cost in EES and TES by reducing the revenue in peak load period.

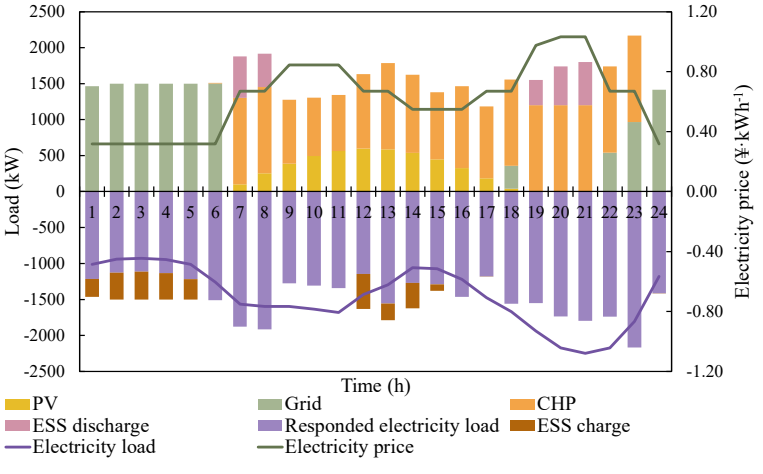

Fig. 6 Electricity balance in transition season

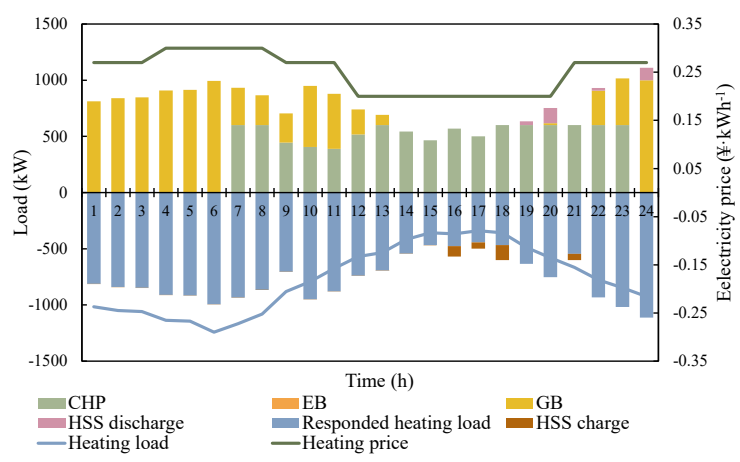

Fig. 7 Electricity balance in transition season

\section{Conclusion}

A bi-level planning method for energy storage system of integrated community energy system considering demand response is proposed in this paper. Compared with the conventional planning method, The following conclusions are drawn:

1) The peak load is be reduced through demand response. The energy bill of the energy consumer is decreased.

2) The investment cost of the energy storage system is reduced by the proposed planning method. The demand response of the energy consumer is utilized by the energy server as an energy storage system to reduce the investment cost of the electricity energy storage and thermal energy storage.

\section{Acknowledgement}

This work was financially funded by the Science and Technology Foundation of State Grid Shandong Electric Power Company (5206071800D9).

\section{Reference}

1. J. Wu, J. Yan, H. Jia, N. Hatziargyriou, N. Djilali, and H. Sun, "Integrated Energy Systems," Applied Energy, vol. 167, pp. 155-157, (2016).

2. B. P. Koirala, E. Koliou, J. Friege, R. A. Hakvoort, and P. M. Herder, "Energetic communities for community energy: A review of key issues and trends shaping integrated community energy systems," 
Renewable and Sustainable Energy Reviews, vol. 56, pp. 722-744, (2016).

3. P.-H. Li and S. Pye, "Assessing the benefits of demand-side flexibility in residential and transport sectors from an integrated energy systems perspective," Applied Energy, vol. 228, pp. 965-979, (2018).

4. H. Alharbi and K. Bhattacharya, "Stochastic Optimal Planning of Battery Energy Storage Systems for Isolated Microgrids," IEEE Transactions on Sustainable Energy, vol. 9, no. 1, pp. 211-227, (2018).

5. W. Kim, J. Shin, and J. Kim, "Operation Strategy of Multi-Energy Storage System for Ancillary Services," IEEE Transactions on Power Systems, vol. 32, no. 6, pp. 4409-4417, (2017).

6. S. Bahramirad, W. Reder, and A. Khodaei, "Reliability-Constrained Optimal Sizing of Energy Storage System in a Microgrid," IEEE Transactions on Smart Grid, vol. 3, no. 4, pp. 2056-2062, (2012).

7. K. Baker, G. Hug, and X. Li, "Energy Storage Sizing Taking Into Account Forecast Uncertainties and Receding Horizon Operation," IEEE Transactions on Sustainable Energy, vol. 8, no. 1, pp. 331-340, (2017).

8. C. Chen, H. Sun, X. Shen, Y. Guo, Q. Guo, and T. Xia, "Two-stage robust planning-operation cooptimization of energy hub considering precise energy storage economic model," Applied Energy, vol. 252, p. 113372, (2019).

9. B. Shen, G. Ghatikar, Z. Lei, J. Li, G. Wikler, and P. Martin, "The role of regulatory reforms, market changes, and technology development to make demand response a viable resource in meeting energy challenges," Applied Energy, vol. 130, pp. 814-823, (2014).

10. C. Bartusch and K. Alvehag, "Further exploring the potential of residential demand response programs in electricity distribution," Applied Energy, vol. 125, pp. 39-59, (2014).

11. S. Bahrami and A. Sheikhi, "From Demand Response in Smart Grid Toward Integrated Demand Response in Smart Energy Hub," IEEE Transactions on Smart Grid, vol. 7, no. 2, pp. 650-658, (2016).

12. L. Huang, J. Walrand, and K. Ramchandran, "Optimal demand response with energy storage management," in 2012 IEEE Third International Conference on Smart Grid Communications (SmartGridComm), (2012), pp. 61-66. 